\title{
Stability and Accuracy of Exponential Extrapolation Method
}

\author{
Asma A. Elbeleze \#, Bachok M. Taib * \\ Unvirsiti Sains Islam Malaysia, Faculty of Science and Technology, 71800 Nilai, Malaysia \\ E-mail:\#elbeleze@yahoo.com, bachok@usim.edu.my
}

\begin{abstract}
The stability of a numerical methods is important consideration that must be considered when solving ODEs, where Since the extrapolation methods are best away to get a high-accuracy solution of ODEs. In this paper we studied the stability and accuracy of the exponential extrapolation method which is in the form of $y=A e^{\alpha h}$ where $A$ and $\alpha$ are constant to be determined using least square fitting. We gave example to explain the accuracy of this method and we explained that the stability region of exponential extrapolation method always inside the $|1+z| \leq 1$.
\end{abstract}

Keywords - Euler's method; exponential extrapolation method; order of accuracy; extrapolation methods; stability region.

\section{INTRODUCTION}

Since the numerical solution that we got using numerical methods sometimes not satisfactory because, the results no accurate, so we need to extrapolate this solution to get high accuracy.

There are variety of approaches available for extrapolate the numerical solution of initial value problems, including polynomial extrapolation, rational extrapolation and exponential extrapolation (that we defined it later in this paper).

Some authors studied the accuracy, stability and derivation of polynomial and rational extrapolation, some overviews of these methods can be find it in [5], [6]. Reference [2] review two solvers of ODEs (Trapezoidal and GBS) and compered their stability domains. Also, they explained how stability depends on the order of extrapolation.

The stability of numerical methods is important consideration that must be considered when solving ODEs. The stability of a particular application can be depend on three factors: the differential equation, the numerical method, and the step size.

In sight into the step size required for stability can be examined by studying a very simple ODE

$$
y^{\prime}=\lambda y
$$

On the other hand, the accuracy may be low for three different reasons: (a) Integrating for a very long time (b) Using a very large step size (c) Using low order method.

In the present paper we introduce the stability and accuracy of exponential extrapolation method. Finally we summarized the main results that have been reached about stability and accuracy for this method.

\section{PRELIMINARY CONSIDERATIONS}

We write the initial value problem in ordinary differential equation as

$$
y^{\prime}=f(x, y), \quad y\left(x_{0}\right)=y_{0}
$$

where $y(x, h)$ is a suitable defined approximation to the exact value $y(x)$ obtained by Euler' method

\section{A. Truncation Error}

Truncation error at any point $x_{k}$ can be classified into

\section{1) Global Error}

The global error is the difference between computed solution and true solution $\mathrm{y}(\mathrm{x})$

$$
e_{k}=y_{k}-y\left(x_{k}\right)
$$

\section{2) Local Error:}

The local error made in one step of numerical method.

$$
l_{k}=y_{k}-u_{k}\left(x_{k}\right)
$$

where $u_{k}\left(x_{k}\right)$ is the solution at previous point $\left(x_{k}, y_{k}\right)$

\section{B. Euler's Method and its Stability Domain}

The Euler's method uses to find the solution and advance it from $y_{n}$ to $y_{(n+1)}$ by taking a set of $n$ steps each of size $h$. The Euler's method can be summarized as,

$$
\begin{gathered}
y_{0}=y\left(x_{0}\right) \\
y_{n+1}=y_{n}+h f\left(x_{n}, y_{n}\right), n=0,1, \ldots, n-1
\end{gathered}
$$

Compared the computed solution $y_{n}$ to the exact value $y(x)$, we obtain 


$$
y_{n}-y(x)=\sum_{j=1}^{\infty} C_{j} h^{j}
$$

Stability domain of Euler's method is obtained by considering the numerical scheme applied to the simple linear ODE (1) For each ODE solver applied to (1), we similarly obtain a certain domain in a complex $z=\lambda h$ plane for which solutions will not grow.

Now, the Euler's scheme applied to (1) is

$$
\begin{aligned}
y_{n+1} & =y_{n}+h \lambda y_{n} \\
& =(1+h \lambda) y_{n}
\end{aligned}
$$

If the absolute value of $(1+h \lambda)$ is greater than unity, the solution will grow in an unbounded fashion. So clearly, the stability depends on the step size $\mathrm{h}$.

The equation (5) can rewrite it as

$$
y_{n+1}=(1+z) y_{n}
$$

The stability domain is inside the circle $|1+z|<1$. The figure 1 explains the stability area of Euler's method.

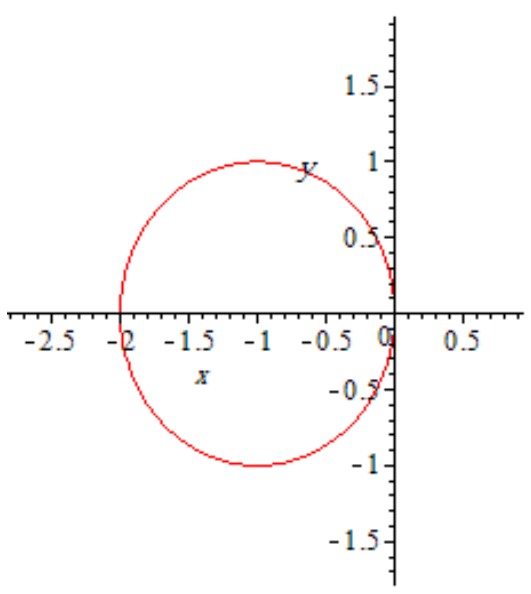

Fig. 1 A stability region of Euler's method

\section{THE EXPONENTIAL EXTRAPOLATION METHOD}

The idea of exponential extrapolation is to obtain a sequence of approximations $\mathrm{T}\left(\mathrm{h}_{1}, \mathrm{x}\right), \mathrm{T}\left(\mathrm{h}_{2}, \mathrm{x}\right), \ldots$ for a given sequence of step sizes $\mathrm{h}_{1}>\mathrm{h}_{2}>\mathrm{h}_{3}>\cdots$, where $h_{j}=\frac{H}{n_{j}}, \mathrm{H}$ is a basic step.

Consider a base numerical method to solve (2) is Euler's method denotes by $y_{h}(x)$. Different numerical solutions $T_{j}^{(0)}$ at $x_{0}+H$ are obtained by applying $n_{j}$ steps of the base method with step size $h_{j}$

$$
T_{j}^{(0)}=y_{h}\left(x_{0}+H\right)
$$

Let us suppose that the global error of the order $p$ for base method employed in (6) has an asymptotic expansion of the form

$$
y(x)-y_{h}(x)=e_{p}(x) h^{p}+\ldots+E_{N} h^{N}+E_{h}(x) h^{N+1}
$$

where $e_{i}(x)$ are errors that independent of $\mathrm{h}$, and $E_{h}$ is bounded $x_{0}<x<x_{f}$ where $x_{f}$ is the last value of $x$.

The algorithm of exponential extrapolation based on Euler's method is formed

$$
T\left(h_{j}, x\right)=A e^{\alpha h_{j}}, \quad j=0,1,2, \ldots
$$

where $\mathrm{A}$ and $\alpha$ are constant to be determine, the best fit of them is by using least square fitting. The numerical scheme (6) and (7) is called exponential extrapolation method.

The extrapolation tableau is

$\begin{array}{ccccc}h_{0} & T_{0}^{(0)} & & & \\ h_{1} & T_{1}^{(0)} & T_{0}^{(1)} & & \\ h_{2} & T_{2}^{(0)} & T_{1}^{(1)} & T_{0}^{(2)} & \\ h_{3} & T_{3}^{(0)} & T_{2}^{(1)} & T_{1}^{(2)} & T_{0}^{(3)} \\ \vdots & \vdots & \vdots & \vdots & \vdots\end{array}$

Each $T_{i j}$ in the above extrapolation tableau is a discrete approximation of $\mathrm{y}(H)$.

\section{Stability domain of exponential extrapolation method}

In above section we have introduced the exponential extrapolation method for the solution of initial value problems in ODEs. Here we discussion the stability of this method.

Since exponential extrapolation method based on Euler's method. So it is the basic one order version of exponential extrapolation method where

$$
y_{n+1}=y_{n}+h f\left(x_{n}, y_{n}\right)
$$

where $y_{n+1}$ is the approximation that is accepted at $\mathrm{x}_{\mathrm{n}+1}$.

Applying (1) in (2) with $y_{0}=1$ gives

$$
\begin{aligned}
y_{1} & =1+h \lambda=1+z \\
y_{2} & =1+h \lambda+h \lambda(1+h \lambda)=1+2 z+z^{2} \\
y_{3} & =1+h \lambda+h \lambda\left(1+2 h \lambda+h^{2} \lambda^{2}\right) \\
& =1+3 z+3 z^{2}+z^{3}
\end{aligned}
$$

So

$$
\begin{aligned}
& y_{1}=1+z \\
& y_{2}=1+2 z+z^{2} \\
& y_{3}=1+3 z+3 z^{2}+z^{3}
\end{aligned}
$$

For non-growing solution $|1+z| \leq 1$ this implies $\operatorname{Real}(\lambda) \leq 0$. Thus, tracing $\mathrm{z}=\lambda \mathrm{h}$ in complex $\mathrm{z}$-plane for $\mathrm{y}=\mathrm{e}^{\mathrm{i} \theta}, \theta \in[0,2 \pi]$.

From above expressions, we can say that stability domains of exponential extrapolation method always take the same area of the Euler's method.

In general we have

$$
y_{N}=(1+z)^{N}
$$

So the area stability of exponential extrapolation is inside the circle.

\section{ACCURACY OF EXPONENTIAL EXTRAPOLATION METHOD}

Accuracy refers to how closely a computed value agrees with the true value. Truncation error is dominant factor determining accuracy of numerical solution of ODEs. The order of accuracy of numerical method is $p$ if

$$
l_{k}=O\left(p_{k}{ }^{k+1}\right)
$$

When we mention the order of accuracy for particular numerical method, we usually mean the order of the global truncation error. 
Determine accuracy of numerical method by comparing exact and numerical solution. In addition, local error estimate is needed to ensure that desired accuracy is achieved.

\section{A. Order accuracy of Euler's method}

Here we explain how to determine the accuracy of Euler's method. The technique is then applicable to higher-order methods. Let us consider we have eq 1 and

$$
y_{n+1}=(1+\lambda h) y_{n}
$$

Assuming $y\left(x_{0}\right)=1$ and $N$ is the number of steps, we have

$$
y_{N}=(1+\lambda h)^{N}
$$

Let $x=\xi=N . h$. We use Taylor series to expand the exact solution

So

$$
e^{\lambda \xi}=1+\lambda \xi+\frac{(\lambda \xi)^{2}}{2}+\frac{(\lambda \xi)^{3}}{3}+\ldots
$$

$$
\begin{aligned}
\text { Error }= & y_{N}-e^{\lambda \xi}=(1+\lambda h)^{N}-e^{\lambda \xi} \\
= & 1+N \lambda h+\frac{N(N-1)}{2}(\lambda h)^{2}+\ldots \\
& -\left(1+\lambda \xi+\frac{(\lambda \xi)^{2}}{2}+\ldots\right. \\
& =-\left(\frac{\lambda^{2} \xi}{2}\right) h+\ldots
\end{aligned}
$$

Thus, we arrive at well-known first order global accuracy $O(h)$ for Euler's method.

Now, we will apply the technique mentioned above to determine accuracy of exponential extrapolation method. When we applied Taylor expansion on exponential extrapolation method with respect to the step size $h$ we reached to that the global accuracy of it has one order accuracy.

\section{NUMERICAL EXPERIMENT}

To study the accuracy of exponential extrapolation method we are given a simple ODE $y^{\prime}=-y$ which has the exact solution $\mathrm{y}=\mathrm{e}^{-\mathrm{x}}$, with step size $\mathrm{h}=0.1$. The results are shown in Table1. There, the error and the value of $T(h, x)$ for the method are tabulated
TABLE 1.

The VALUE OF $T(H, X)$ AND THE ERROR FOR $\mathrm{Y}^{\prime}=-\mathrm{Y}$

\begin{tabular}{|l|l|l|}
\hline Step size & \multicolumn{2}{|l|}{ Exponential extrapolation } \\
\hline $\mathrm{H}$ & $T(h, x)$ & The error \\
\hline 0.05 & 0.9025 & $2.3 \times 10^{-3}$ \\
\hline 0.025 & 0.904877344 & $3.9 \times 10^{-5}$ \\
\hline 0.0125 & 0.904837038 & $3.8 \times 10^{-7}$ \\
\hline 0.00625 & 0.904837419 & $1.9 \times 10^{-7}$ \\
\hline
\end{tabular}

In above table we noticed that for higher orders of accuracy the error of Exponential extrapolation method is decrease with decrease of the step sizes.

\section{CONCLUSIONS}

In this paper we have presented briefly the exponential extrapolation method. Also we explained that the stability region of this method is inside the circle $|1+\mathrm{z}|<1$, because it dependent on Euler's method as the basic method. On the other hand, we noticed that the accuracy of this method increase with decrease the step size (where step size tending to zero). So, the exponential extrapolation would only gain one order of accuracy.

\section{REFERENCES}

[1] Asma A. Elbeleze, Bachok M. Taib, Exponential Extrapolation Method for the Solution of IVP in ODEs, in ICMSA 2011, paper $p$. 89.

[2] Avram Sidi, Practical Extrapolation Methods Theory and Applications, Cambridge university press(2003).

[3] Bengt F.,Julia Z. and Jongwoo L., "Stability and accuracy of time extrapolated ADI-FDTD methods for Solving Wava Equations", Journal of computational and Applied Mathematics, vol.200, pp. 178-192, 2007.

[4] Bulirsch,R., and J. Stoer, Numerical Treatement of Ordinary Differential Equations by Extrapolaion Methods, Numerisch Mathematik,8,pp.1-13 (1966).

[5] E. Hairer and G.Wanner, Solving Ordinary Differential Equations II: Stiff and Differential-Algebraic Problems, Springer (1993).

[6] Lambert J. D., Computational Methods in Ordinary Differential Equations, J. Wiley and Sons (1973).

[7] Lambert J. D., Stiffness In Computational Techniques for Ordinary Differential Equations,I. Gladwell and D. K. Sayers, Eds. Academic Press, Orlando, Fla (1980).

[8] Oprea N. and Marginean D., Application of extrapolation methods to initial value problems in ordinary differential equations. pp 10311038, "Petru Maior" University of Targu-Mure (2009). 